\title{
Unilateral-dominant reduction in muscle volume in female knee osteoarthritis patients: computed tomography-based analysis of bilateral sides
}

\author{
Ayumi Tsukada ${ }^{1}$, Kentaro Uchida ${ }^{1,2^{*}}$, Jun Aikawa ${ }^{1}$, Shotaro Takano ${ }^{1}$, Dai Iwase ${ }^{1}$, Manabu Mukai ${ }^{1}$,
} Masayuki Miyagi ${ }^{1}$, Yuta Nanri ${ }^{3}$, Gen Inoue ${ }^{1}$ and Masashi Takaso ${ }^{1}$

\begin{abstract}
Background: Muscle weakness is associated with osteoarthritis pathology. A recent study demonstrated that measuring muscle volume using computed tomography (CT)-based analysis and comparing bilateral muscles in the same patient allowed for accurate evaluation of muscle volume in unilateral hip osteoarthritis (OA) patients. Here, we evaluated muscle volume using CT-based analysis and compared bilateral muscles in knee OA (KOA) patients.

Methods: CT images were obtained from 35 female radiographic KOA patients the day prior to total knee replacement surgery. Muscle volume (MV) was semi-automatically analyzed. Knee extension muscle strength (MS) was determined using a hand-held dynamometer. The severity of KOA patients' clinical symptoms was examined using four domains of the Japanese Orthopedic Association (JOA) score. We compared the difference in MS ( $\triangle M S)$ and MV ( $\Delta M V)$ between the operated side (OS), which exhibited severe radiographic OA or severe pain, and the contralateral side (CS).

Results: JOA score was significantly lower in the OS than CS. MV and MS were also significantly lower in the OS than CS. There was no correlation between MV and MS or between MV and MS as a percentage of body weight on either side. However, $\triangle \mathrm{MV}$ was positively correlated with $\triangle \mathrm{MS}$ and pain on walking in the JOA.

Conclusions: We evaluated MV and MS using bilateral CT images of the legs of KOA patients. A reduction in MV was observed on the OS, and was correlated with a reduction in MS and pain on walking. Bilateral CT image analysis may be useful for evaluating the relationship between OA pathology and muscle atrophy.
\end{abstract}

Keywords: Computed tomography, Bilateral, Muscle volume, Muscle strength, Osteoarthritis

\section{Background}

Knee osteoarthritis (KOA) is a well-known musculoskeletal disorder and a key cause of disability, particularly in elderly individuals [1]. A number of biomechanical pathways likely play major roles in KOA. For example, excessive

\footnotetext{
* Correspondence: kuchida@med.kitasato-u.ac.jp

'Department of Orthopedic Surgery, Kitasato University School of Medicine,

1-15-1 Minami-ku Kitasato, Sagamihara City, Kanagawa 252-0374, Japan

${ }^{2}$ Shonan University of Medical Sciences Research Institute, Nishikubo 500, Chigasaki City, Kanagawa 253-0083, Japan

Full list of author information is available at the end of the article
}

mechanical stress arising from either a reduction in loadbearing area on the surface of a joint or applying a heavy load can halt the repair of damaged joint tissue [2]. Muscle weakness is a key measure of the extent of disability in OA patients [3]. A number of reports have demonstrated that a reduction in lean mass in the lower limbs is common in OA patients [4], and this reduction is correlated with a heightened risk of falls [5-7]. Therefore, accurate evaluation of the muscles of $\mathrm{KOA}$ patients is important to better

C C The Author(s). 2020 Open Access This article is licensed under a Creative Commons Attribution 4.0 International License, which permits use, sharing, adaptation, distribution and reproduction in any medium or format, as long as you give appropriate credit to the original author(s) and the source, provide a link to the Creative Commons licence, and indicate if changes were made. The images or other third party material in this article are included in the article's Creative Commons licence, unless indicated otherwise in a credit line to the material. If material is not included in the article's Creative Commons licence and your intended use is not permitted by statutory regulation or exceeds the permitted use, you will need to obtain permission directly from the copyright holder. To view a copy of this licence, visit http://creativecommons.org/licenses/by/4.0/ The Creative Commons Public Domain Dedication waiver (http://creativecommons.org/publicdomain/zero/1.0/) applies to the data made available in this article, unless otherwise stated in a credit line to the data. 
understand the relationship between the muscle and $\mathrm{OA}$ pathology.

Previous studies have proposed that translational studies that fill the gap between basic and clinical research using three-dimensional imaging data provide a basis for creating anatomical models of the human anatomy and may allow orthopedic surgeons to determine ideal practices before orthopedic surgery [8-10]. Computed tomography $(\mathrm{CT})$ is used for various applications, including the evaluation of orthopedic tumors [11] and fractures [12], and preoperative planning of orthopedic surgery [13]. CT is also regularly used to quantify muscle volume (MV) at regions around the knee and hip [14-16]. In particular, the cross-sectional area (CSA) of the affected region determined from $\mathrm{CT}$ images is measured to evaluate MV [14-16]. However, these measurements vary widely and are dependent on the location at which the section is taken. In addition, muscle mass varies greatly from person to person due to differences in height or body mass index (BMI), making it difficult to conduct comparisons and accurately elucidate the effect of KOA on muscle mass. A recent study showed that muscle volume in unilateral hip OA patients could be accurately evaluated by measuring muscle volume using CT-based analysis and comparing bilateral muscles in the same patient [17].

Here, we evaluated muscle volume using CT-based analysis and conducted comparisons between bilateral muscles in KOA patients.

\section{Methods}

The study protocol received ethics approval from the Institutional Review Board (IRB) for Clinical Research and Treatment of Kitasato University (IRB approval number: B20-133).

According to power analysis conducted with $\alpha=0.05$ and power $=0.80$ using G*POWER3, 34, and 17 samples were needed to obtain a statistically significant difference in muscle strength (MS) and MV, respectively, between the operated and contralateral sides. Therefore, we obtained CT images from 35 female patients with radiographic KOA the day prior to total knee replacement surgery. Four domains of the Japanese Orthopedic Association (JOA) score, namely, pain on walking (domain I), pain on ascending or descending stairs (domain II), range of motion (domain III), and joint effusion (domain IV) [18], were used to evaluate symptom severity (Table 1).

\section{Muscle strength}

Knee extension MS was determined using a hand-held dynamometer ( $\mu$ Tas F-1; Anima, Tokyo) as participants were seated on a chair with their hips and knees flexed at $90^{\circ}$. Knee extension MS was expressed as the raw value in Newtons $(\mathrm{N})$ and as a percentage of body
Table 1 The Japanese Orthopedic Association (JOA) score for patients with knee osteoarthritis

\section{Pain on walking (total 30 points)}

Walking $1 \mathrm{~km}$ or more usually with no pain, without regard for mild pain, or rarely feeling pain with certain activities

Walking $1 \mathrm{~km}$ or more regardless of pain

Walking $500 \mathrm{~m}$ or more, but less than $1 \mathrm{~km}$ without regard for pain

Walking $100 \mathrm{~m}$ or more, but less than $500 \mathrm{~m}$ without regard for 15 pain

Walking indoors or nearby, but less than $100 \mathrm{~m}$ without regard $\quad 10$ for pain

Inability to walk

Inability to stand

II. Pain on ascending or descending stairs (total 25 points)

No pain

Pain with handrails, but no pain with step-by-step ambulation

Pain relieved by using handrails

Pain with step-by-step ambulation, pain relieved by using handrails

Pain even with step-by-step ambulation and handrail use

Inability to ascend or descend because of pain

10

III. Range of motion (total 35 points)

Squatting 35

Sideways or cross-legged sitting $\quad 30$

Flexion or arc of motion of $110^{\circ}$ or more $\quad 25$

Flexion or arc of motion of $75^{\circ}$ or more $\quad 20$

Flexion or arc of motion of $35^{\circ}$ or more $\quad 10$

Flexion or arc of motion less than $35^{\circ}$ including ankylosis or 0 severe flexion contracture

IV. Joint effusion (total 10 points)

No edema, no swelling 10

Occasional puncture required 5

Frequent puncture required 0

This table was translated by the authors with permission from the Japanese Orthopedic Association

weight (\% BW). The difference in MS $(\Delta \mathrm{MS})$ between the operated side (OS), which exhibited severe radiographic OA or severe pain, and the contralateral side (CS) was calculated using the following equation:

Difference in muscle strength $(\Delta \mathrm{MS}, \%)$

$$
=\left(\mathrm{MS}_{\mathrm{OS}} / \mathrm{MS}_{\mathrm{CS}}-1\right) \times 100
$$

\section{CT-based analysis of femoral muscle volume}

Axial CT images were used to measure patients' femoral MV. CT images comprising a $10-\mathrm{mm}$ region of interest were taken $200 \mathrm{~mm}$ above the knee joint at 1-mm thickness and semi-automatically analyzed using the MIMI 

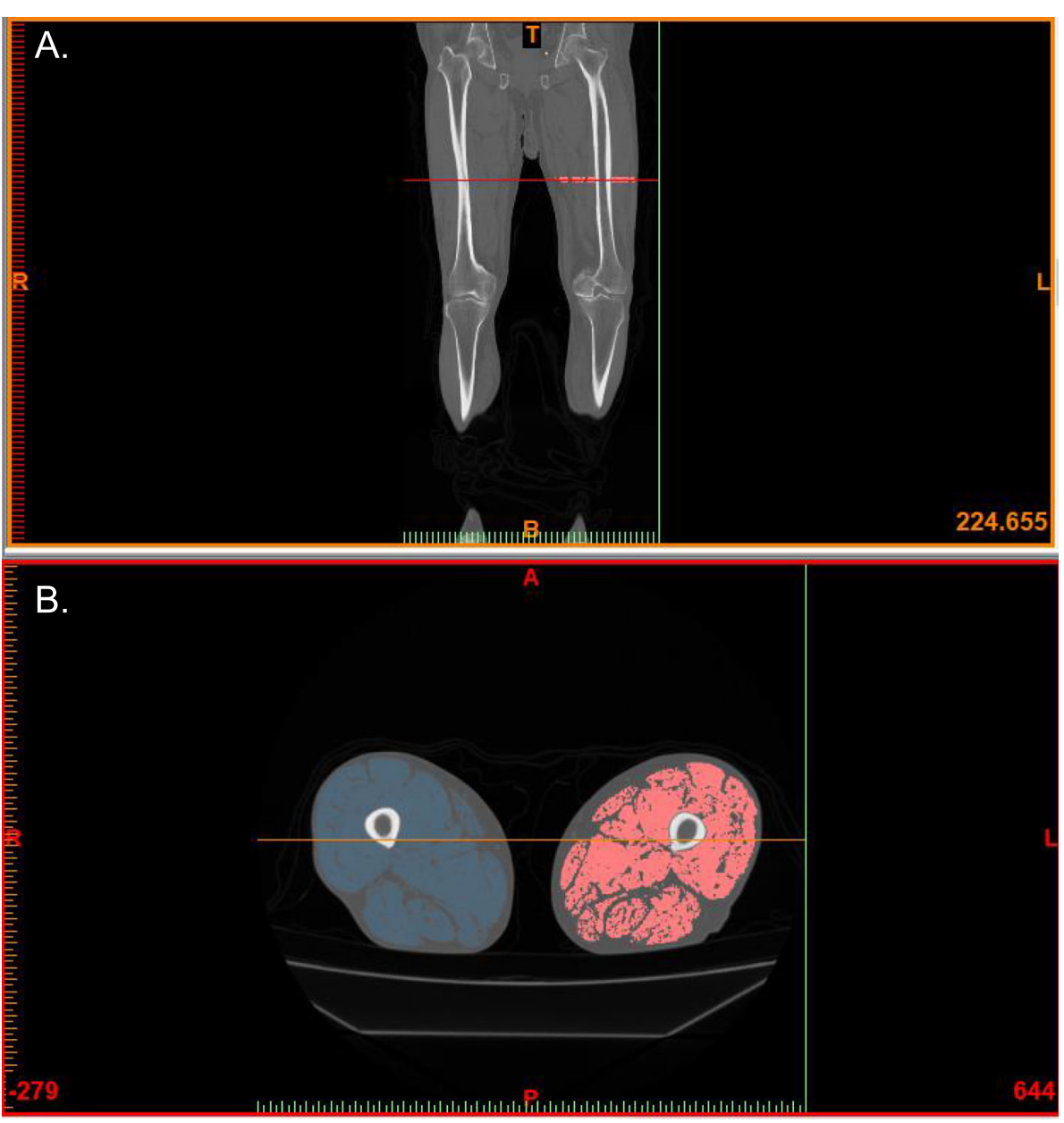

Fig. 1 Estimation of muscle volume using CT-image analysis. a CT image of both legs of a patient. Red line indicates the location of a digital slice taken approximately $200 \mathrm{~mm}$ above the knee joint. $\mathbf{b} C T$ images of a cross section of bilateral muscles were semi-automatically analyzed in a 10$\mathrm{mm}$ region of interest $(\mathrm{ROI})$ using the $\mathrm{MIMICS}^{\circledR}$ software

Table 2 Patient demographic data

\begin{tabular}{|c|c|c|c|}
\hline Parameter & Operated side & Contralateral side & $P$ value \\
\hline Age (years) & $72.4 \pm 7.4$ & & \\
\hline Height (cm) & $149.0 \pm 6.0$ & & \\
\hline Weight (kg) & $57.9 \pm 10.4$ & & \\
\hline $\mathrm{BMI}\left(\mathrm{kg} / \mathrm{m}^{2}\right)$ & $26.1 \pm 4.1$ & & \\
\hline$K / L$ grade $(1,2,3,4), N$ & $0,0,6,29$ & $2,4,15,14$ & $P=0.127$ \\
\hline \multicolumn{4}{|l|}{ JOA score } \\
\hline Total & $54.6 \pm 10.6^{*}$ & $73.4 \pm 16.3$ & $P<0.001$ \\
\hline Pain on walking & $16.3 \pm 5.9^{*}$ & $21.6 \pm 7.3$ & $P<0.001$ \\
\hline Pain on ascending or descending stairs & $6.0 \pm 4.8^{*}$ & $14.4 \pm 7.9$ & $P<0.001$ \\
\hline ROM & $25.7 \pm 3.4^{*}$ & $27.9 \pm 0.7$ & $P=0.001$ \\
\hline Joint effusion & $6.6 \pm 3.8^{*}$ & $9.6 \pm 1.4$ & $P<0.001$ \\
\hline
\end{tabular}

Data indicate mean \pm standard deviation unless otherwise indicated

$B M I$ body mass index, $K / L$ Kellgren/Lawrence grade, $J O A$ Japanese Orthopedic Association, ROM range of motion

${ }^{*} P<0.05$ versus contralateral side 
Table 3 Muscle strength and muscle volume

\begin{tabular}{|c|c|c|c|c|}
\hline & Operated side & Contralateral side & Difference (\%) & $P$ value \\
\hline Muscle strength $(N)$ & $0.52 \pm 0.22^{*}$ & $0.61 \pm 0.25$ & $-10.14 \pm 25.18$ & $P=0.001$ \\
\hline$\%$ Muscle strength to body weight (\% BW) & $28.78 \pm 9.71^{*}$ & $33.62 \pm 11.57$ & & $P=0.001$ \\
\hline Muscle volume $\left(\mathrm{cm}^{3}\right)$ & $77.95 \pm 13.11^{*}$ & $83.03 \pm 12.09$ & $-6.0 \pm 8.9$ & $P=0.001$ \\
\hline
\end{tabular}

Data indicate mean \pm standard deviation unless otherwise indicated

${ }^{*} P<0.05$ versus contralateral side

$\mathrm{CS}^{\bullet}$ software (Materialise Japan Co., Ltd., Yokohama, Japan) (Fig. 1). Femoral MV was compared between OS and CS. Differences in MV between OS and CS were calculated using the following equation:

Difference in muscle volume $(\Delta \mathrm{MV}, \%)$

$$
=\left(\mathrm{MV}_{\mathrm{OS}} / \mathrm{MV}_{\mathrm{CS}}-1\right) \times 100
$$

\section{Statistical analysis}

Differences between OS and CS were examined using paired $t$ test. The relationship between MV and MS was evaluated using Spearman's correlation coefficient. A $P$ value of $<0.05$ was considered statistically significant. Statistical analysis was performed using the SPPSS software (Version 25.0; SPSS, IBM, Armonk, NY, USA).

\section{Results}

\section{Study participant's demographic information}

The participants' demographic and clinical information are provided in Table 2. Participants' mean age was 72.4 \pm 7.4 years and body mass index was $26.1 \pm 4.1 \mathrm{~kg} / \mathrm{m}^{2}$. There was no difference in the ratio of Kellgren/ Lawrence $(\mathrm{K} / \mathrm{L})$ grades between the OS and CS. Total JOA score and the score for all four domains (pain on walking, pain on ascending or descending stairs, range of motion, and joint effusion) were significantly lower in the OS than CS.

\section{Muscle strength and volume}

MS and MS as a percentage of body weight were significantly reduced in the OS compared to CS $(P=0.001$; Table 3). MV was also significantly reduced in the OS compared to CS $(P=0.001$; Table 3$)$. No correlation was found between MV and MS (Fig. 2a) or MV and MS as a percentage of body weight on the OS (Fig. 2b). However, $\triangle \mathrm{MV}$ was positively correlated with $\triangle \mathrm{MS}$ on the OS $(r=0.651, P<0.001$; Fig. 2c).

Relationship between clinical score, muscle strength, and muscle volume

There was no correlation between the total JOA score and the score for three domains (pain on ascending or descending stairs, range of motion, and joint effusion) and $\triangle \mathrm{MS}$ (Fig. 3a, c-e) or $\Delta \mathrm{MV}$ (Fig. 4a, c-e). In contrast, both $\triangle \mathrm{MS}$ and $\triangle \mathrm{MV}$ were significantly correlated with domain I (pain on walking) in JOA ( $\Delta \mathrm{MS}, r=0.342, P=0.044$, Fig. $3 \mathrm{~b} ; \Delta \mathrm{MV}, r=0.375$, $P=0.026$; Fig. 4b).

\section{Discussion}

Previous studies have shown that KOA patients exhibit a reduction in muscle mass in the lower limbs compared to age-matched healthy controls $[6,19,20]$. However, a cohort study reported a weak association between bone mass, measured based on bioimpedance analysis (BIA) using a body composition analyzer, on muscle strength in KOA patients [19]. Consistent with previous reports,

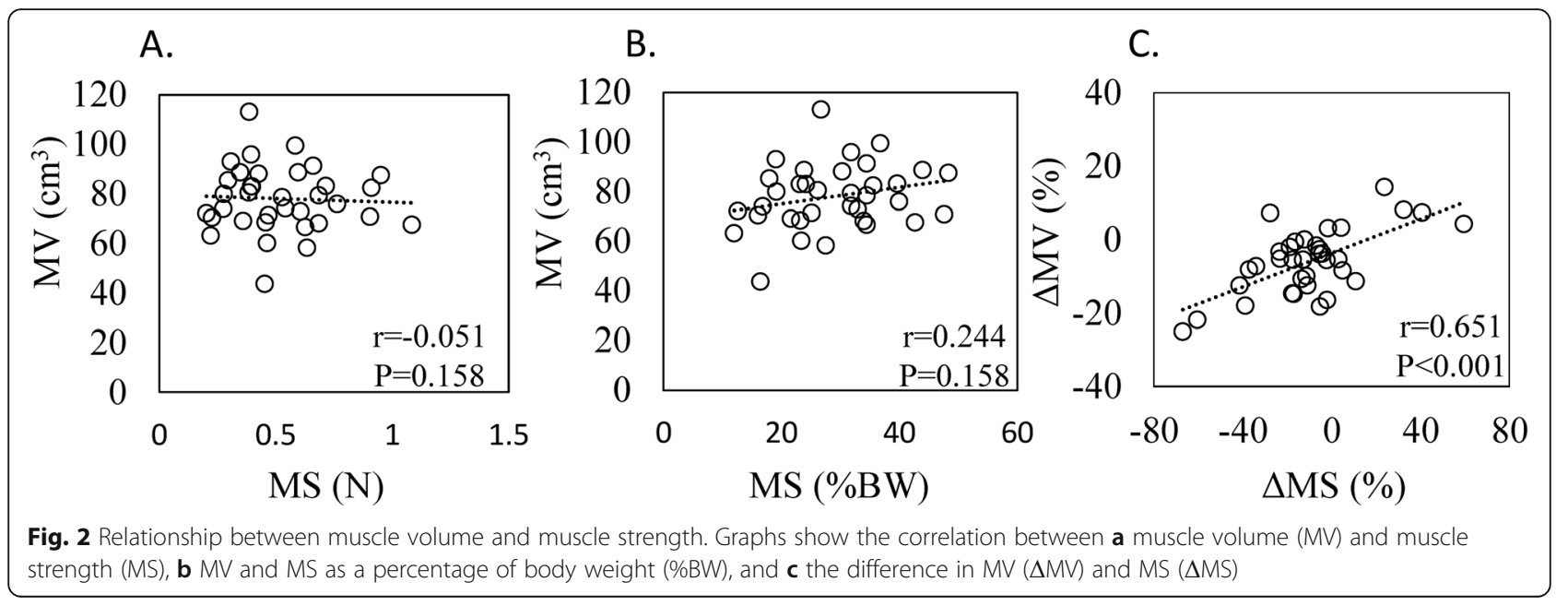



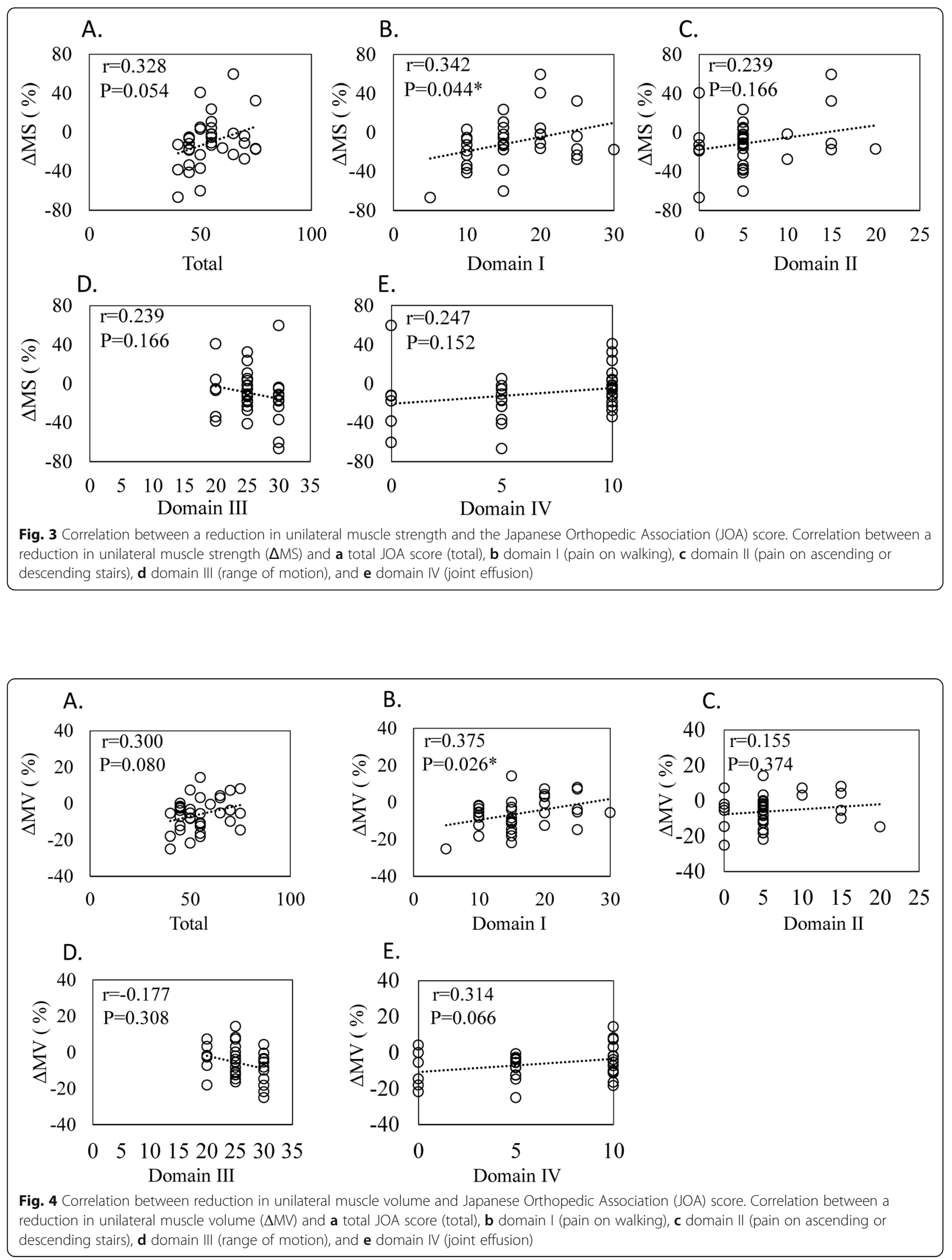
Table 4 Advantages and limitations of this study

\begin{tabular}{|c|c|}
\hline Advantages & Limitations \\
\hline $\begin{array}{l}\text { - CT-based analysis of bilateral muscle volume accurately reflects muscle } \\
\text { strength in patients with KOA. } \\
\text { - CT-based analysis of bilateral muscle volume enables exclusion of parame- } \\
\text { ters such as age and BMI, which affect muscle mass and strength. }\end{array}$ & $\begin{array}{l}\text { - The analyzed muscle comprises several different muscles, including } \\
\text { the rectus femoris, biceps femoris, and adductor longus. } \\
\text { - This study was a cross-sectional study. } \\
\text { - Patients with bilateral knee osteoarthritis were included among the } \\
\text { participants. }\end{array}$ \\
\hline
\end{tabular}

$C T$ computed tomography, KOA knee osteoarthritis, $B M I$ body mass index

we found no correlation between MS and MV. However, bilateral MV analysis using CT images taken from KOA subjects showed a unilateral decrease in MV on the side with severe OA and that this reduction was strongly correlated with a reduction in MS. CT-based analysis of bilateral sides may therefore be a useful tool for evaluating muscle weakness in KOA patients due to the exclusion of parameters such as age and BMI, which affect muscle mass and strength (Table 4).

There is a logical biomechanical explanation for the association between leg muscle mass and knee symptoms. Periarticular muscles, which keep injured and degenerated knees structurally stable and supported, with greater mass relative to total body mass provide better stability, resulting in less pain. Several studies examining changes to the muscles in the lower limbs have shown that low impact exercises like swimming and cycling are beneficial for reducing pain in patients with KOA [21, 22]. Therefore, low impact therapeutic approaches may be important for relieving pain in KOA patients.

Several reports have suggested that unilateral mechanical unloading is a cause of muscle reduction [23-25]. Studies in humans have reported that, as a result of unilateral lower limb unloading, there is a $7 \%$ decrease in muscle CSA after 21 days [24] and a 16\% decrease after 35 days $[23,25]$. In the present study, MV was reduced by approximately $6 \%$ on the side with severe OA, and this reduction was associated with pain on walking. Underuse of the muscle due to pain may therefore explain the unilateral reduction in MV in KOA patients.

This study has several limitations (Table 4). First, the analyzed muscle comprises several different muscles, including the rectus femoris, biceps femoris, and adductor longus. Assessment of individual muscles is necessary to obtain a practical understanding of functional disability in patients with KOA. Second, our study was a crosssectional study. Longitudinal studies are needed to clarify the relationship between OA development and muscle reduction. Finally, patients with bilateral KOA were included among the participants in this study.

\section{Conclusion}

We evaluated MV using bilateral CT images of the legs of patients with severe knee pain. MV was reduced on the side with severe $\mathrm{OA}$ and this reduction was correlated with a decrease in MS and pain. Bilateral CT image analysis may be useful for evaluating the link between OA and muscle pathology due to the ability to exclude parameters such as age and BMI, which affect muscle mass and strength. Further investigation using a longitudinal study may clarify the relationship between OA development and muscle reduction.

\section{Abbreviations}

BIA: Bioimpedance analysis; BMI: Bone mass index; CSA: Cross-sectional area; CS: Contralateral side; CT: Computed tomography; IRB: Institutional Review Board; JOA: Japanese Orthopedic Association; OA: Osteoarthritis;

OS: Operated side; \%BW: Percentage of body weight; MV: Muscle volume; MS: Muscle strength

Acknowledgements

We thank Ms. Yuko Onuki for her help with CT image analysis.

\section{Authors' contributions}

AT, KU, and MT designed the study and analyzed the data. AT and KU wrote the manuscript. JA, ST, DI, MM (Mukai), MM (Miyagi), YN, and GI participated in data collection, analysis, and interpretation. All authors read and approved the final manuscript.

\section{Funding}

This study was supported in part by research grants from the Parents' Association of Kitasato University School of Medicine.

\section{Availability of data and materials}

The datasets supporting the conclusions of this article are included within the article. The raw data can be requested from the corresponding author.

\section{Ethics approval and consent to participate}

All experimental protocols were approved by the Ethics Review Board of Kitasato University (Permission number: B20-133).

\section{Consent for publication}

Not applicable.

\section{Competing interests}

The authors declare that they have no competing interests.

\section{Author details}

${ }^{1}$ Department of Orthopedic Surgery, Kitasato University School of Medicine, 1-15-1 Minami-ku Kitasato, Sagamihara City, Kanagawa 252-0374, Japan. ${ }^{2}$ Shonan University of Medical Sciences Research Institute, Nishikubo 500, Chigasaki City, Kanagawa 253-0083, Japan. ${ }^{3}$ Department of Rehabilitation, Kitasato University Hospital, 1-15-1 Kitasato, Minami-ku, Sagamihara, Kanagawa 252-0375, Japan.

Received: 30 September 2020 Accepted: 5 November 2020 Published online: 19 November 2020

\section{References}

1. Brandt KD, Dieppe P, Radin E. Etiopathogenesis of osteoarthritis. Med Clin North Am. 2009;93(1):1-24 xv.

2. Heidari B. Knee osteoarthritis prevalence, risk factors, pathogenesis and features: Part I. Caspian J Intern Med. 2011;2(2):205-12. 
3. Fisher NM, White SC, Yack HJ, Smolinski RJ, Pendergast DR. Muscle function and gait in patients with knee osteoarthritis before and after muscle rehabilitation. Disabil Rehabil. 1997;19(2):47-55.

4. Toda Y, Kobayashi T. The usefulness of walking for preventing sarcopenia in dieting postmenopausal women complaining of knee pain. Ann N Y Acad Sci. 2000;904:610-3.

5. Conroy MB, Kwoh CK, Krishnan E, Nevitt MC, Boudreau R, Carbone LD, Chen $H$, Harris TB, Newman AB, Goodpaster BH. Muscle strength, mass, and quality in older men and women with knee osteoarthritis. Arthritis Care Res (Hoboken). 2012;64(1):15-21.

6. O'Reilly SC, Jones A, Muir KR, Doherty M. Quadriceps weakness in knee osteoarthritis: the effect on pain and disability. Ann Rheum Dis. 1998;57(10): 588-94.

7. Segal NA, Glass NA, Torner J, Yang M, Felson DT, Sharma L, Nevitt M, Lewis CE. Quadriceps weakness predicts risk for knee joint space narrowing in women in the MOST cohort. Osteoarthritis Cartilage. 2010;18(6):769-75.

8. Mediouni M. A new generation of orthopaedic surgeons: "T-model". Curr Orthop Pract. 2019;30(5):444-5.

9. Mediouni M, Schlatterer DR, Madry H, Cucchiarini M, Rai B. A review of translational medicine. The future paradigm: how can we connect the orthopedic dots better? Curr Med Res Opin. 2018;34(7):1217-29.

10. Mediouni M, Madiouni R, Gardner M, Vaughan N. Translational medicine: challenges and new orthopaedic vision (Mediouni-Model). Curr Orthop Pract. 2020;31(2):196-200

11. Mediouni M, Schlatterer DR. Orthopaedic tumors: what problems are we solving, and are universities and major medical centers doing enough? J Orthop. 2017;14(2):319-21.

12. Konda SR, Goch AM, Leucht P, Christiano A, Gyftopoulos S, Yoeli G, Egol KA. The use of ultra-low-dose CT scans for the evaluation of limb fractures: is the reduced effective dose using $C T$ in orthopaedic injury (REDUCTION) protocol effective? Bone Joint J. 2016;98-B(12):1668-73.

13. Atesok K, Galos D, Jazrawi LM, Egol KA. Preoperative planning in orthopaedic surgery: current practice and evolving applications. Bull Hosp Jt Dis (2013). 2015;73(4):257-68.

14. Arokoski MH, Arokoski JP, Haara M, Kankaanpaa M, Vesterinen M, Niemitukia LH, Helminen HJ. Hip muscle strength and muscle cross sectional area in men with and without hip osteoarthritis. J Rheumatol. 2002;29(10):2185-95.

15. Ikeda S, Tsumura H, Torisu T. Age-related quadriceps-dominant muscle atrophy and incident radiographic knee osteoarthritis. J Orthop Sci. 2005; 10(2):121-6.

16. Rasch A, Bystrom AH, Dalen N, Berg HE. Reduced muscle radiological density, cross-sectional area, and strength of major hip and knee muscles in 22 patients with hip osteoarthritis. Acta Orthop. 2007;78(4):505-10.

17. Momose T, Inaba Y, Choe H, Kobayashi N, Tezuka T, Saito T. CT-based analysis of muscle volume and degeneration of gluteus medius in patients with unilateral hip osteoarthritis. BMC Musculoskelet Disord. 2017;18(1):457

18. Okuda M, Omokawa S, Okahashi K, Akahane M, Tanaka Y. Validity and reliability of the Japanese Orthopaedic Association score for osteoarthritic knees. J Orthop Sci. 2012;17(6):750-6.

19. Muraki S, Akune T, Teraguchi M, Kagotani R, Asai Y, Yoshida M, Tokimura F Tanaka S, Oka H, Kawaguchi $\mathrm{H}$, et al. Quadriceps muscle strength, radiographic knee osteoarthritis and knee pain: the ROAD study. BMC Musculoskelet Disord. 2015;16:305.

20. Slemenda C, Heilman DK, Brandt KD, Katz BP, Mazzuca SA, Braunstein EM, Byrd D. Reduced quadriceps strength relative to body weight: a risk factor for knee osteoarthritis in women? Arthritis Rheum. 1998;41(11):1951-9.

21. Alkatan M, Baker JR, Machin DR, Park W, Akkari AS, Pasha EP, Tanaka H. Improved function and reduced pain after swimming and cycling training in patients with osteoarthritis. J Rheumatol. 2016;43(3):666-72.

22. Al-Khlaifat L, Herrington LC, Hammond A, Tyson SF, Jones RK. The effectiveness of an exercise programme on knee loading, muscle cocontraction, and pain in patients with medial knee osteoarthritis: a pilot study. Knee. 2016;23(1):63-9.

23. Dudley GA, Duvoisin MR, Adams GR, Meyer RA, Belew AH, Buchanan P. Adaptations to unilateral lower limb suspension in humans. Aviat Space Environ Med. 1992;63(8):678-83.

24. Schulze K, Gallagher P, Trappe S. Resistance training preserves skeletal muscle function during unloading in humans. Med Sci Sports Exerc. 2002; 34(2):303-13.

25. Hather BM, Adams GR, Tesch PA, Dudley GA. Skeletal muscle responses to lower limb suspension in humans. J Appl Physiol (1985). 1992;72(4):1493-8.

\section{Publisher's Note}

Springer Nature remains neutral with regard to jurisdictional claims in published maps and institutional affiliations.
Ready to submit your research? Choose BMC and benefit from:

- fast, convenient online submission

- thorough peer review by experienced researchers in your field

- rapid publication on acceptance

- support for research data, including large and complex data types

- gold Open Access which fosters wider collaboration and increased citations

- maximum visibility for your research: over $100 \mathrm{M}$ website views per year

At BMC, research is always in progress.

Learn more biomedcentral.com/submissions 